\title{
Interactive comment on "Towards simplification of hydrologic modeling: identification of dominant processes" by S. L. Markstrom et al.
}

\author{
S. Höllering (Referee) \\ simon.hoellering@kit.edu
}

Received and published: 18 February 2016

\section{General comments}

The authors presented an interesting idea of a methodological framework wherein parameters of the HRU based Precipitation-Runoff Modeling System (PRMS) can be identified as influencial in terms of essential hydrological model based processes and statistical streamflow indices serving as objective functions. Parameter influence on model output was evaluated by parameter senstivity index values originating from global sensitivity analysis with the Fourier Amplitude Sensitivity Test (FAST). The approach aims at reducing the number of model input parameters to focus on conceptualised processes assumed as hydrologically relevant within the watersheds of the conterminous United States. 
I generally agree with the concept of referencing model response functioning in form of derived objective functions with dependent partial parameter sensitivities for region specific model parameter identification. This is one of the aspects which would be really worth publishing.

Apart from that, fundamental assumptions underlying this study are not sufficiently clarified to address the discussed issues effectively, which are certainly topical and relevant for model based catchment hydrology. The paper is technically well-structured, exhibiting findings of the presented concept concisely but it lacks the required presentation quality at too many different points. However, I found some serious shortcomings and recommend to revise a number of major and minor specific and technical points before the manuscript can be reconsidered for publication.

\section{Specific comments}

What is the main purpose of your paper?

You mention a number of issues e.g. "parameter identification", "process identification", "calibration advise for modelers" or "identification of [model] structural inadequacies". A better focus on one or two of these issues, preferably on the first and second is advisable here. As uncertainty analysis is not the issue here, I furthermore suggest to remove the part starting from $\mathrm{P} 16 \mathrm{~L} 29$, which is also rather speculative.

Please also name your assumptions more precisely!

The fundamental assumption of this study is, that the conceptualisation of PRMS is structurally adequate to reproduce all hydrological processes of the CONUS. It is however not adressed, whether this assumption is valid or not or if the study doesn't 
claim to be transferable to real world processes and consequently stays a pure virtual PRMS experiment. Conclusions on the dominant hydrological processes are only valid if it is shown that PRMS actually is a good representation of hydrological processes. Processes in the study purely originate from and are defined by the PRMS structure whereby a comparison with observational data might be helpful in this application to show potential deficiencies or justify the fundamental assumption.

Interactive

P2L19/P10L20: As you similarly found out, more complex processes such as the reproduction of streamflow and its components as well as mountainous regions require more calibration parameters. The general rather small remaining subset of sensitive parameters explaining the majority of the model output variance of processes might be predefined by the conceptual structure of PRMS and a hint to overparameterization. The number of parameters required in a process is also predetermined by the model/process concept and its complexity. Maybe be a bit more specific and less general or sketchy in stating your findings i.e. in the sense of the influence a parameter exerts on a process which might not be purely predetermined by the concept of a model.

P3L13: (How) do these two aspects of complexity correspond to the ones stated in the abstract and explained directly above these lines? Maybe you should be more precise here!

P3L32: This issue has also been partly discussed e.g. by Reusser and Zehe (2011).

P5L8: HRUs are purely derived and defined by their geographic and topographic Printer-friendly version location. Process identification and catchment classification might be hampered by this definition e.g. by mingling of processes leading to a complex interplay and location specific response behaviour which cannot be always captured by one HRU. In 
addition to your discussed points a redefinition of HRUs based on dominant hydrologic processes instead of the applied discretisation based on geographic position might be a conceivable outcome and a consequence of your study maybe helpful for calibration.

P5L20: Here a more precise explanation might be helpful. Is simulated streamflow at locations with stream gauges evaluated differently from streamflow at sites without observations?

P7L1: Here more attention to further studies with streamflow indices could be given (see e.g. Yadav et al. (2007)). Please discuss your choice in some more details.

P9L25: I suggest to start this chapter with the sentence "To identify the expected count of parameters ... (P9L28)" first the theory, then a specific example.

P10L23/P13L8: This view might be kind of model structure/concept specific (as stated above) and is not surprising as streamflow is a convolution of these individual processes. Isn't total HRU runoff in PRMS the pure product or sum of the other streamflow processes (surface runoff, interflow and baseflow), hence involved process parameters add up to a larger number suggesting more complexity? Maybe you can be a bit more precise in the explanations (P13L13).

P15L25: To my knowledge PRMS offers different modules for PET calculations. (How) do sensitivity results and parameter identificaton change by replacing one module by another? This might be subject of future studies and worth mentioning.

P16L3: Someone who is interested in modelling the selected catchment is probably better advised to have a look at historical meteorological observations. From these it should be obvious that snowmelt might not be of any interest here. 


\section{Technical corrections}

Typing errors:

The spelling and writing needs improvement and proofreading. To mention several of them:

Please be consistent in the writing and consider HESS manuscript preparation guidelines for authors e.g. Figure, Fig.

P2L15: indicate instead of indicates

P4L3/P16L14: watersheds

P8L15: Here poor comprehensibility can be better avoided by changing three to seven objective functions: "... 56 combinations of three objective functions and eight processes (plus totals)."

P11L7: “...is surprising...”

P15L12: "This is probably because it is a major component of the hydrologic cycle that is..."

P15L21: than

P16L7: used

P16L11: "...is defined..."

P16L14: processes

Reference/citation errors:

Citations in the manuscript are correct while the year 2014 in complete reference is not:

Markstrom, S. L., Regan, R. S., Hay, L. E., Viger, R. J., Webb, R. M. T., Payn, R. A., and LaFontaine, J. H.: PRMS-IV, the precipitation-runoff modeling system, version 4, U.S. Geological Survey Techniques and Methods, book 6, chap. B7, 158, http://dx.doi.org/10.3133/tm6B7, 2015 
Figures:

General remarks:

Resolution and quality of the presented figures and maps seem to be generally not high enough or pixelated and need substantial improvement. Unfortunately, the labeling of latitudinal and longitudinal lines are not readable at all. Please improve the legibility or remove it or incorporate it in only one figure which might be enough to show it once. Some of the shortcomings are listed here:

Figure 1: This map lacks both sufficient quality and a valuable information content. In my oppinion a different form of presentation such as histograms or kernel density estimates for selected attributes of HRUs could be beneficial.

Figure 2: Please use consistent spelling or abbreveations for objective functions across tables and figures. Please explain the additional column "Process average" in the results section 4.1 and the meaning of the legend.

The caption should also provide more information.

Figure 3: Better use as Figure 1. It furthermore contains little information and poor legibility of region names.

Figure 4: "The plots A-H summarize..."

Figure 5: Please clarify the connection to the ordered listing of Table 1.

Figure 6: Please raise font sizes of titles above each map to be readable or remove them from the figure.

\section{References}

Reusser, D. E. and Zehe, E.: Inferring model structural deficits by analyzing temporal dynamics of model performance and parameter sensitivity, Water Resources Research, 47, doi:10.1029/2010WR009946, 2011.

Printer-friendly version

Yadav, M., Wagener, T., and Gupta, H.: Regionalization of constraints on expected watershed response behavior for improved predictions in ungauged basins,

Discussion paper 
Advances in Water Resources, 30, 1756-1774, doi:10.1016/j.advwatres.2007.01.005, 2007.

Interactive comment on Hydrol. Earth Syst. Sci. Discuss., doi:10.5194/hess-2015-508, 2016.

Interactive

comment 